\title{
Proyecto METEOESCUELA. Una red de observación escolar en Cantabria
}

https://doi.org/10.31978/639-19-010-0.795

\author{
José Luis Arteche García ${ }^{1}$ (jartecheg@aemet.es) \\ María Rosa Pons Reynés ${ }^{1}$ (mponsr@aemet.es) \\ Margarita Garvia Polo' (mgarviap@aemet.es) \\ Aurora Ortega González ${ }^{1}$ (aortegag@aemet.es) \\ Eroteida Sánchez García ${ }^{1}$ (esanchezg@aemet.es) \\ Policarpo Martínez Fernández ${ }^{1}$ (pmartinezf@aemet.es)
}

${ }^{1}$ AEMET / Delegación Territorial en Cantabria

\begin{abstract}
RESUMEN
Dentro de una tradición de muchos años desarrollando un gran esfuerzo divulgativo del tiempo y el clima surgió el proyecto educativo METEOESCUELA, desarrollado por la Delegación Territorial de AEMET en Cantabria con la colaboración de la Consejería de Educación, Cultura y Deporte de Cantabria. El objetivo del proyecto es la creación de una red de observación meteorológica escolar, cuyos datos se comparten en un portal web (https://meteoescuela.aemet.es/cantabria). Todo ello pensado y dirigido al alumnado de quinto y sexto de Educación Primaria y de todos los cursos de Educación Secundaria Obligatoria. El proyecto se puso en marcha en el curso académico 2015-2016 y en la actualidad cuenta ya con la participación de 33 centros escolares, con una vocación de seguir creciendo a través de nuevas incorporaciones en los próximos cursos.

El proyecto pretende fomentar el interés de la población escolar por la meteorología y la climatología mediante diferentes actividades: los alumnos realizan la observación meteorológica de temperatura, precipitación y meteoros, suben los datos a la web, pueden descargarlos para trabajar en el aula y participan en la elaboración de un Catálogo de Nubes y Meteoros enviando sus fotografías. La web proporciona también una serie de recursos educativos para el profesorado en temas de meteorología y climatología y se incluyen noticias divulgativas actualizadas sobre cambio climático, predicción, observaciones de un determinado episodio y otros temas de interés para el proyecto.
\end{abstract}

PALABRAS CLAVE: red escolar; educación; divulgación; portal web; cambio climático.

\section{INTRODUCCIÓN}

La Delegación Territorial de AEMET en Cantabria lleva más de treinta años participando en acciones de divulgación de la meteorología en el ámbito escolar, principalmente a través de las visitas escolares a la Delegación pero también a través de talleres, conferencias, exposiciones y jornadas científicas.

La finalidad del programa METEOESCUELA, el último paso de toda esta trayectoria, se sustenta en los siguientes pilares básicos:

- Dotar a la población escolar de Cantabria de recursos para la enseñanza de la observación meteorológica. El recurso básico es la página web, la cual constituye el corazón de la red de observaciones escolares METEOESCUELA donde los alumnos pueden alojar las observaciones realizadas en el centro escolar. 
- Poner a disposición de los centros de Cantabria datos meteorológicos recogidos en la red METEOESCUELA para la posterior práctica de la climatología. La ventaja de utilizar datos reales y recogidos por ellos mismos dará una nueva dimensión a la hora de afrontar ejercicios sobre climatología en el aula.

- Ofrecer recursos para la didáctica de la meteorología en el aula a los colegios e institutos de Cantabria.

- Fomentar el interés de los alumnos por la meteorología y la climatología.

Como objetivos secundarios del proyecto, podríamos hablar de los siguientes:

- Facilitar al alumno conocimiento acerca de la observación meteorológica y las distintas variables meteorológicas.

- Adquirir hábitos de toma rutinaria y sistemática de datos como elemento de la formación científico-técnica de los alumnos.

- Concienciar sobre el trabajo en red para el estudio de la climatología, mediante las actividades de toma simultánea de datos, intercambio de los mismos, etc.

- Favorecer la conciencia sobre el cambio climático en la población escolar de Cantabria.

- Conocer y contactar con otros alumnos que trabajan en el proyecto METEOESCUELA.

- Conseguir distintas vivencias y experiencias relacionadas con la meteorología.

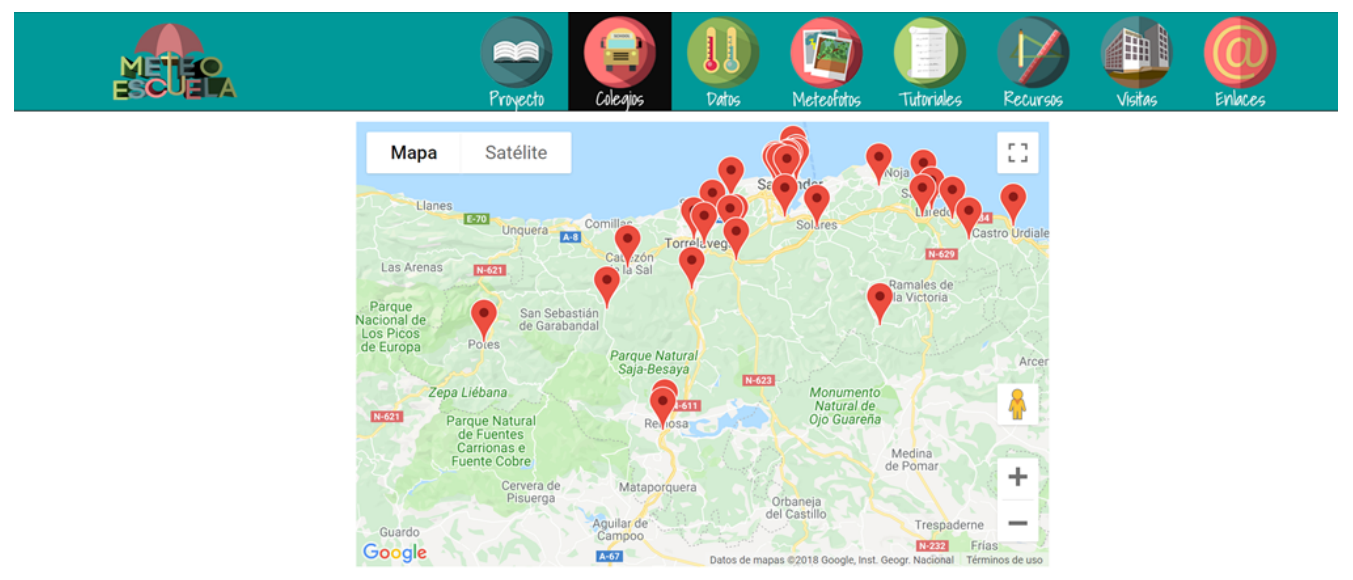

Figura 1. La red METEOESCUELA.

\section{ASUNTOS ORGANIZATIVOS}

La implantación y desarrollo del proyecto METEOESCUELA ha requerido y requiere de un trabajo en equipo, condición indispensable cuando se dispone ya de una red de observatorios escolares amplia. La toma de decisiones es mejor desarrollarla en grupo, a la vez que se establece cada año, tras el verano, un cronograma de acciones y sus responsables para afrontar la campaña que empieza con el curso escolar. Toda la documentación, actas de reuniones, etc. se suben a un repositorio en owncloud para estar a disposición de todos los miembros del grupo.

Cada año se decide el crecimiento de la red, una vez pasado el arranque inicial fuerte de hace unos años, tras sopesar el coste de tiempo y esfuerzo que significa disponer de una red cada vez más amplia de centros escolares adheridos al proyecto. 
Existe un modelo de carta de invitación que se envía a todos los centros a través de los canales de comunicación de la Consejería de Educación, Cultura y Deporte del Gobierno de Cantabria; la colaboración la encontramos en la Dirección General de Innovación Educativa y Centros, que ha apoyado desde el principio el proyecto coincidiendo con la presencia de profesores en los centros escolares tras el verano. Los nuevos miembros del proyecto deberán comenzar a introducir datos en enero.

Para optar a formar parte de METEOESCUELA los centros han de enviarnos unos datos básicos rellenando una ficha que se descarga de la propia página web, con detalles del centro, fotografías, curso que participará, ubicaciones de las que disponen para realizar las observaciones, nombre y contacto del profesor responsable, etc.

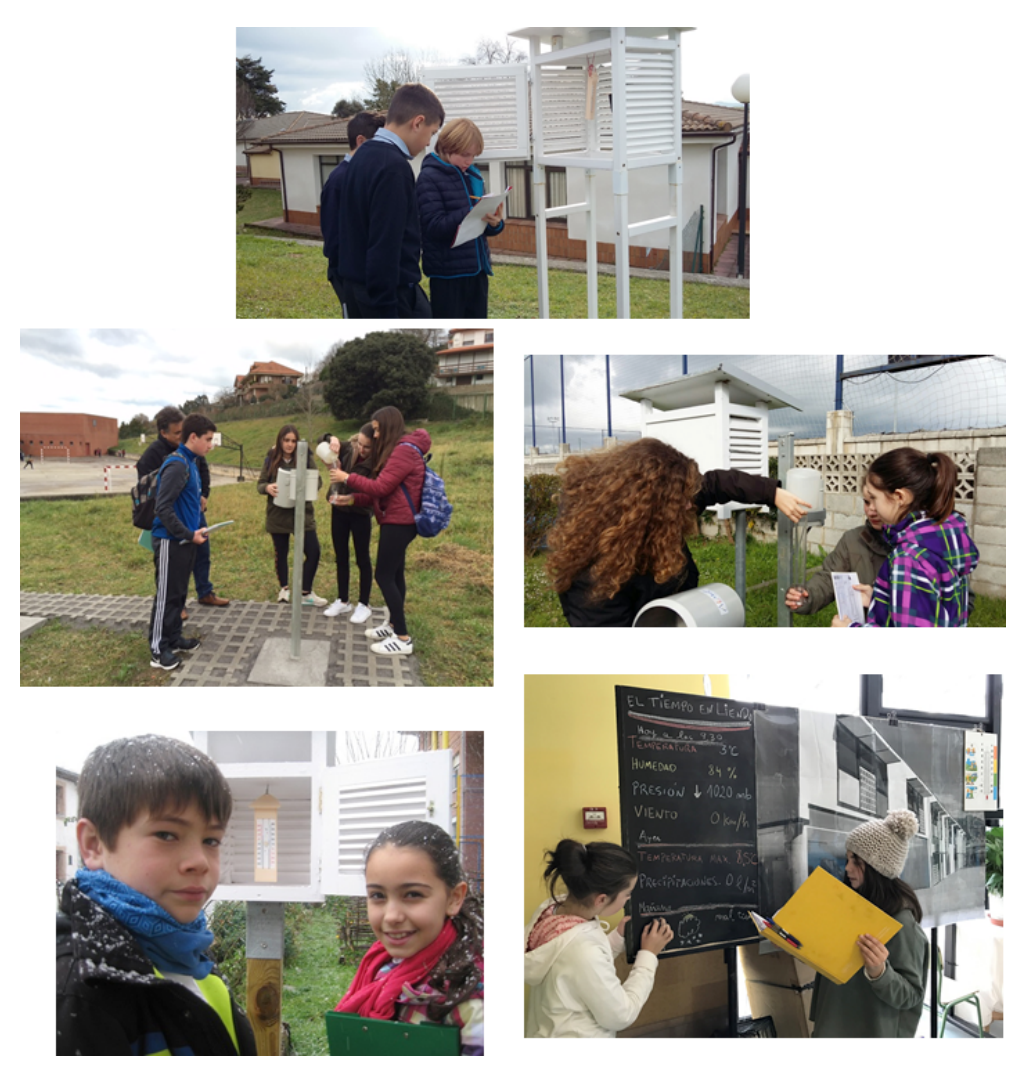

Figura 2. Los alumnos, protagonistas de METEOESCUELA.

Se tiene un encuentro en la delegación territorial con los nuevos responsables del proyecto en los centros seleccionados ese año para formar parte de la red y se establece un plan de visitas lo más rápido posible para visitar personalmente cada centro, elegir la ubicación del punto de observación y entregarles el material de pluviometría (poste, pluviómetro Hellman y probeta) así como un termómetro de máxima y mínima, inicialmente de mercurio y en la actualidad de alcohol. Los pluviómetros provienen de los que se renuevan en la red climatológica de la región, los cuales se sanean y con una mano de pintura «reviven» nuevamente para continuar sirviendo a la Meteorología y la Climatología. Se repasa en dicho encuentro el funcionamiento del portal, se les entrega las claves de acceso, se visita el observatorio de la delegación para que conozcan en qué consiste y cómo se hace la observación meteorológica.

Un aspecto importante a destacar es la elección de los nuevos miembros cada año. Se ha establecido una tabla de baremo en la que se tienen en cuenta una serie de indicadores: dispersión geográfica (cubrir la provincia lo máximo posible, figura 1), pertenecer a zonas de atención educativa preferente (Castro Urdiales, Liébana, Campoo), desarrollar un programa integrado de innovación educativa, ser centro con programas de sostenibilidad y haber quedado en lista de espera en la anterior convocatoria. 
Los centros seleccionados deberán contar con un espacio apropiado para alojar los instrumentos, realizar una observación diaria, subir los datos a la web con una periodicidad diaria o semanal y tener un coordinador del proyecto en dicho centro que será quien reciba una sencilla formación inicial a cargo del personal de AEMET para obtener un rendimiento óptimo del programa. Asimismo, se debe contar con el compromiso del centro educativo de mantener el proyecto activo ante posibles cambios en las personas responsables, garantizando así la continuidad de la actividad. Este constituye un aspecto clave del proyecto METEOESCUELA, pues no debemos olvidar que se trata de una actividad más de las muchas que realizan los centros escolares de la región y las personas cambian de destinos, no solo los profesores que se inician en este proyecto, sino los propios equipos directivos de los centros. Es por ello que hay que estar continuamente alentando la buena marcha de esta iniciativa, haciendo ver que es un trabajo que muestra sus frutos más bonitos a medio y largo plazo, con series largas y muy completas fruto del «Observatorio meteo» del colegio o instituto.

Todas las actuaciones que se llevan a cabo se ponen en conocimiento de la Consejería de Educación pues se desea que ellos sean conocedores plenamente de la marcha del proyecto dada su gran implicación en el mismo.

Se obtuvo desde el principio el compromiso por parte de la Consejería de sufragar cada año la adquisición de unas pequeñas garitas para ubicar los termómetros. Los centros reciben el dinero del coste de las mismas de modo que las adquieran con alguna empresa fabricante.

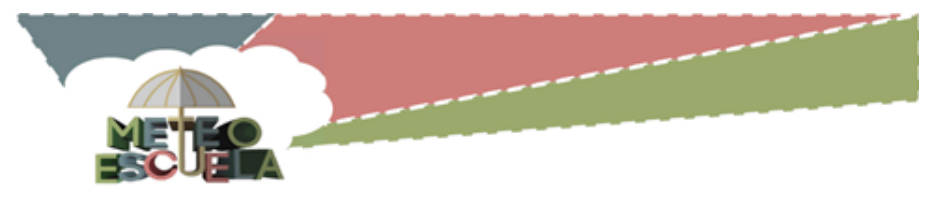

EI IES ELASTILLERO, participa en el proyecto

METEOF SCUELA desde el curso $2017-2018$ con el siguiente: Indicativo Hidrológico Escolar E-1109a 就 $=-1$ EMet

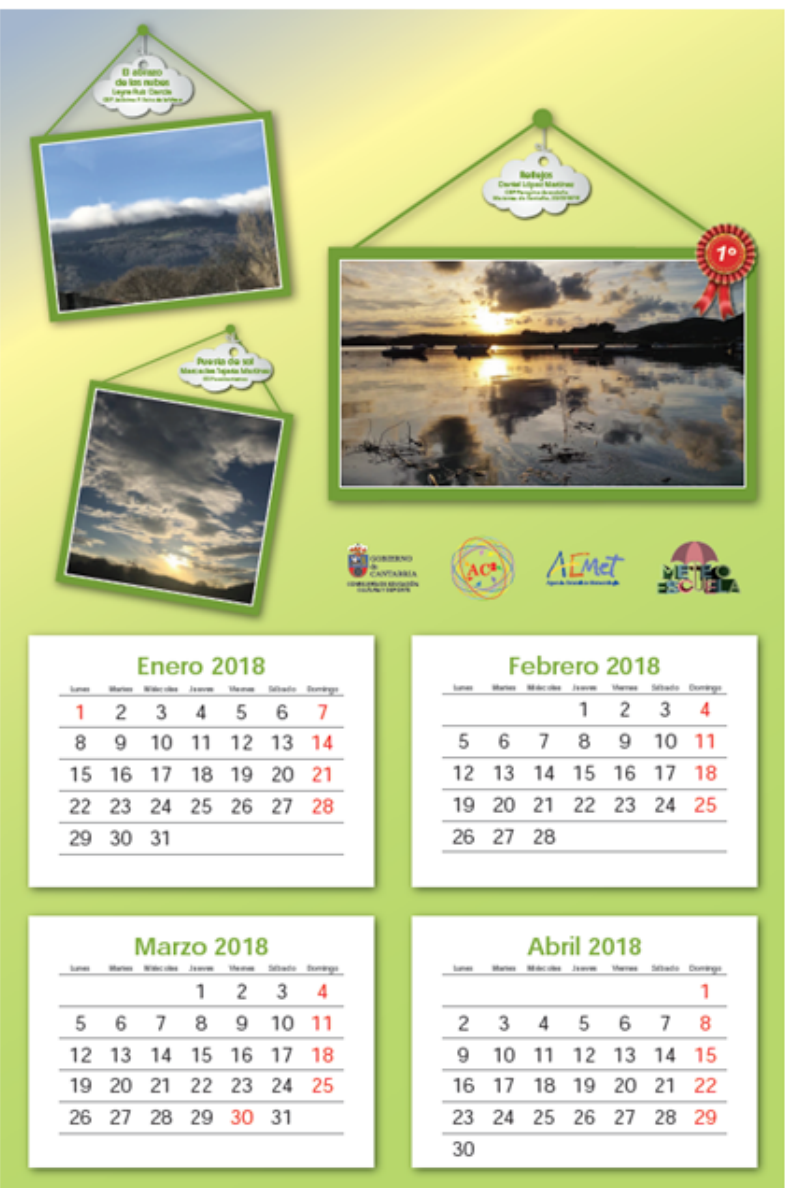

Figura 3. Indicativo hidrológico escolar y calendario escolar de Cantabria.

Siempre se da la posibilidad a cada centro de que puedan ellos mismos fabricar las garitas, con unas breves indicaciones, sufragando en ese caso la Consejería el coste de los materiales necesarios. Ha habido algún caso, es cierto que pocos, en el que ha ocurrido esto.

Una vez que el Centro comienza su andadura le hacemos una visita en la que en un acto simbólico, con todos los alumnos, les entregamos una sencilla placa con el indicativo hidrológico escolar para que lo coloquen en un lugar visible. Este indicativo (figura 3) es único para ese observatorio y se determina por la ubicación geográfica exacta del centro, al igual que cualquier otro observatorio de nuestra red de observación climatológica, siguiendo las normas que marca la Organización Meteorológica Mundial (OMM). 


\section{ESTRUCTURA Y CONTENIDOS DEL PORTAL WEB METEOESCUELA}

\section{https://meteoescuela.aemet.es/}

El acceso a la web es abierto si bien los centros participantes gozan de ciertos privilegios que les permiten la introducción de datos y subir fotografías por medio de permisos en forma de contraseñas, diferentes para los alumnos y los profesores.

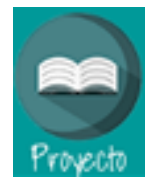

En el apartado «Proyecto» (figura 4) se indica lo que es METEOESCUELA y cómo nació, cómo participar y se informa de otras actividades que se realizan a lo largo del curso escolar: talleres «Meteorólogos por un día», con la presencia siempre de TVE, y «Vigilantes del Tiempo» para los más pequeños. Estos talleres ya se han llevado a cabo en varias ediciones y se dispone de un formato establecido que se intenta reproducir $y$, siempre que se puede, mejorar cada año. Todo se documenta para darles continuidad en el tiempo y que puedan reproducirse fielmente en cualquier momento. Asimismo, cualquier delegación territorial que lo desee puede disponer de dichainformación.

Otras actividades son la presencia en semanas culturales que organizan los centros escolares y en las que se suelen ofrecer exposiciones reducidas pero lo más representativas posible de lo que significa el tiempo y el clima, charlas divulgativas, etc.

Los centros participantes se pueden conocer por una ficha que habla de su ubicación, datos, galería de fotos, etc.: «Colegios».

El enlace «Datos» nos va a permitir visualizar en forma de tablas o de gráficas-climogramas, de forma individualizada por centro, o de forma conjunta, un grupo de ellos, las temperaturas, precipitaciones y meteoros observados durante un día, mes o año.

Cuando se accede a través de las claves propias de acceso se puede realizar la introducción de los datos diarios o mensuales.
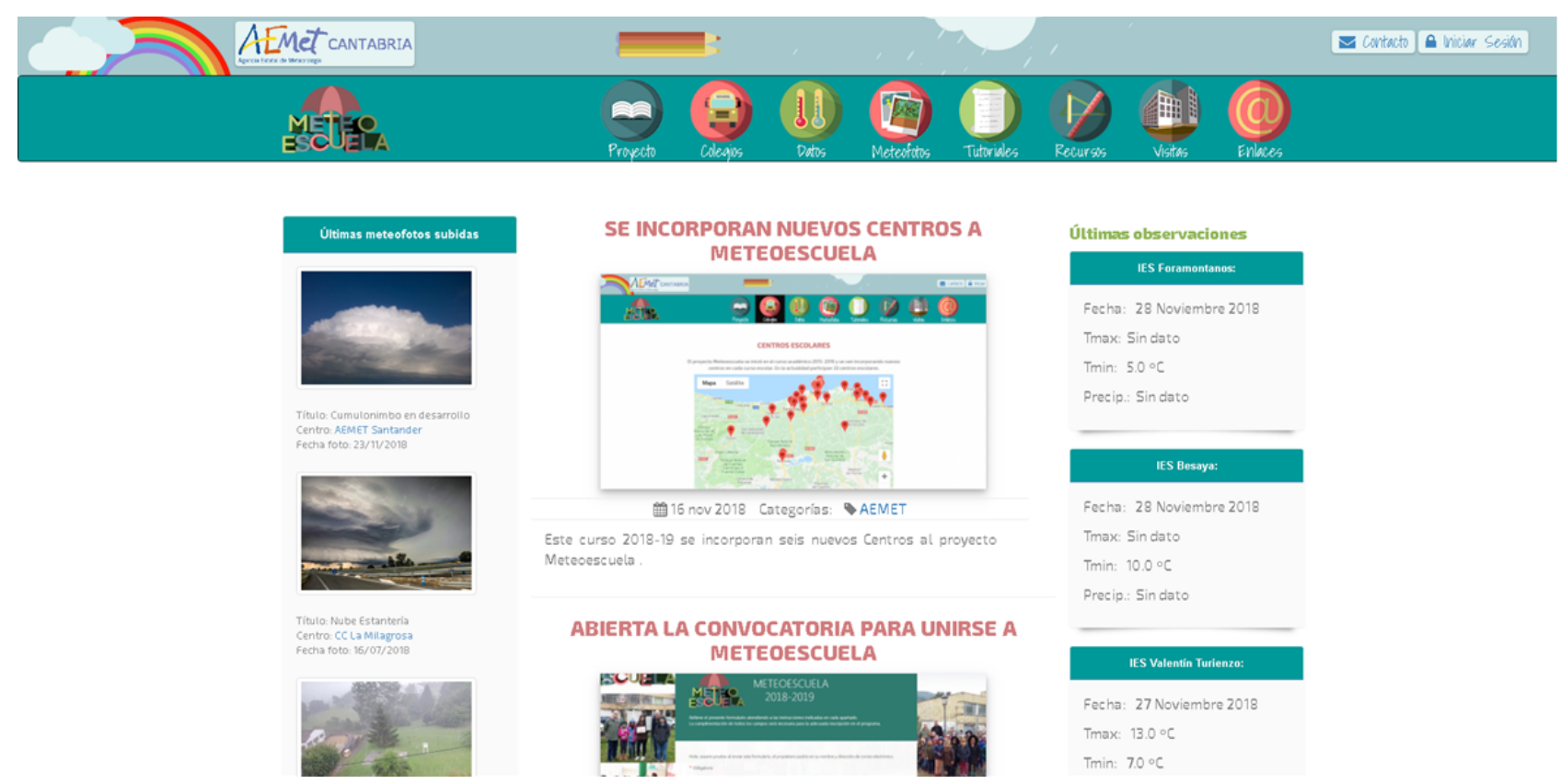

Figura 4. Portada de METEOESCUELA. 
En el apartado «Meteofotos» se anima a los alumnos de los centros a participar enviando fotografías de nubes y de meteoros a lo largo del curso escolar.

Unas Guías de meteoros y de nubes intentan ayudarles en dicho trabajo.

«La instalación de instrumentos», «la observación», «la grabación de datos en el portal», «la consulta y descarga de datos» así como lo relativo a usuarios y permisos en la web se recogen en unos «Tutoriales» de fácil acceso.

Un apartado de «Recursos educativos» está disponible para uso en los centros por parte de los profesores y alumnos.

En este apartado ponemos a disposición de toda la comunidad escolar material educativo de diversa índole y formato (material gráfico, talleres, juegos, pósteres de la OMM) para que se puedan descargar y realizar diversas actividades propuestas.

Se ha etiquetado bajo dos conceptos diferentes:

- Por un lado la idoneidad de los destinatarios, separados por ciclos: Educación Primaria Secundaria, Bachiller y Formación Profesional.

- Por otro la parte de la meteorología que trabajan: observación, climatología, predicción.

Además, en el apartado de «Enlaces» se puede encontrar una sección específica de Recursos educativos disponibles en internet.

Se sugiere la propuesta de algún contenido nuevo o cualquier tipo de sugerencia a través de la cuenta de correo meteoescuela@aemet.es.

Una preciosa y estupenda «Guía para la observación e identificación de nubes» (Francisco Martín León y José Antonio Quirantes) permitirá a los más curiosos adentrarse en el mundo de la observación de las maravillosas nubes, una afición que a quien «engancha» no lo abandona nunca.

En la estructura principal del portal METEOESCUELA se ofrece información de lo que viene siendo desde siempre la actividad de divulgación con más solera en nuestra Delegación Territorial de AEMET, antiguamente Instituto Nacional de Meteorología, las «Visitas» semanales de los centros escolares durante el curso.

En la antigua sede, como Centro Meteorológico en el Paseo del Alta, en Santander, se inició ya hace muchos años un programa de visitas que continúan hoy en día en la actual ubicación de la Delegación Territorial en Cantabria, en Cueto (Santander).

En los últimos años se ha consolidado un programa de visitas didácticas dirigidas a alumnos desde $5^{\circ}$ de Primaria hasta Bachiller. La convocatoria se realiza a través de la Consejería de Educación para llegar a todos los centros educativos.

El calendario de las visitas abarca todos los martes del periodo escolar, en horario de 11 a 13 horas. La visita se estructura sobre la base de «los tiempos de la meteorología»:

- Presente - Observación y vigilancia.

- Pasado-Climatología.

- Futuro-Predicción.

El inicio de la actividad se desarrolla con una breve presentación sobre meteorología, el trabajo que se desarrolla en AEMET y una introducción a la observación, climatología y predicción. 
Se inicia entonces el recorrido didáctico al jardín de observación meteorológico, exposición de instrumentos meteorológicos para una explicación más detallada de su funcionamiento, asistencia al lanzamiento del globo para el sondeo meteorológico — lo que más les gusta a los escolares(12.00 horas o 13.00 horas según el horario de invierno o verano), Rincón del Clima y visita al Grupo de Predicción y Vigilancia de la delegación para conocer las labores de predicción y vigilancia operativas. Algunas visitas se complementan con la realización de experimentos relacionados con la meteorología.

\section{DINAMICA DE TRABAJO EN LOS CENTROS ESCOLARES}

\subsection{La toma de datos diaria}

Cada centro ha de determinar la forma en la que los estudiantes han de organizarse para la toma diaria de los datos de lluvia, temperatura máxima y mínima así como anotar los meteoros observados (se dispone en la página web de un catálogo explicativo con imágenes). Todos somos conscientes de que los chicos y chicas no son observadores profesionales y que tanto la garita como el pluviómetro pueden no estar ubicados en las condiciones ideales que marca la OMM para la toma de datos, pero se intenta hacer las cosas lo más cercano a las condiciones ideales.

Un problema que surgió desde el diseño inicial del proyecto fue cómo resolver la falta de datos de los fines de semana y cortos periodos vacacionales, de hasta cuatro o cinco días, de modo que las series de datos no quedasen «mutiladas» continuamente. Se decidió resolverlo con la ayuda de las estaciones meteorológicas automáticas (EMA) más cercanas a cada centro escolar. Inicialmente se diseñó un programa externo en Visual Basic en el que había que introducir los datos recogidos los lunes y los datos de ayuda de la EMA correspondiente, pero resultaba un tanto engorroso, de modo que a fecha de hoy el portal realiza automáticamente estas acciones, una vez que introducimos nuestros datos: total de lluvia acumulada, temperaturas máxima y mínima registradas (no sabemos inicialmente a qué días corresponden) y directamente el portal calcula y registra en la base de datos los correspondientes a cada día en el que hemos estado ausentes a partir de la EMA más cercana, de forma que nuestros datos «deducidos» sigan el mismo comportamiento que los de la EMA de AEMET utilizada.

\subsection{La subida de los datos al portal}

Tras la toma diaria de datos, lo cual se realiza en papel (con una ficha impresa ya preparada y que se descarga del propio portal), el siguiente paso es subir los mismos a la página web. Esta tarea se puede hacer cada día (esta es la opción que se recomienda) o semanalmente.

Al final de mes se deben introducir los datos resumen mensuales una vez calculado de forma externa, como un ejercicio de cálculo sencillo (máximos valores, mínimos valores, valores medios, fechas, etc.) o bien se deja al sistema que lo haga por su cuenta siempre que se disponga de datos suficientes. Esta misión únicamente la realizaría el profesor.

Existe la opción de modificar datos erróneos, visualizar los datos diarios y mensuales en forma de tabla o en gráficos desplegables (figura 5).

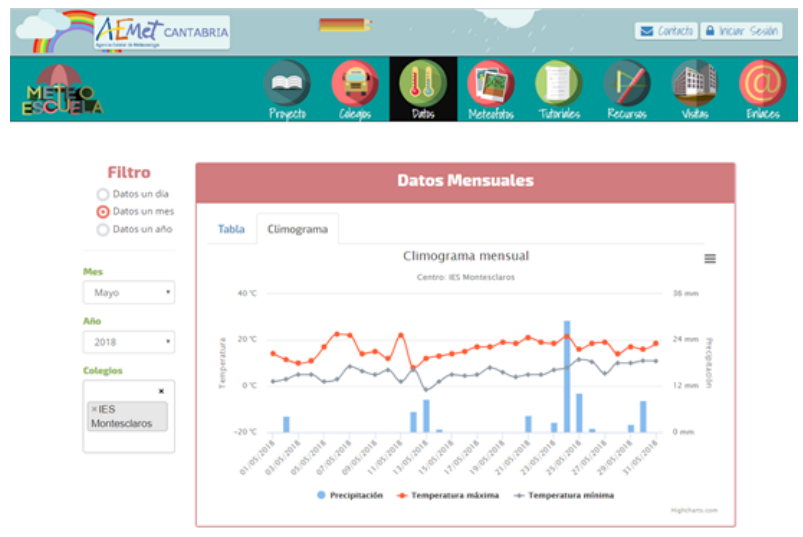

Figura 5. Visualización de datos en METEOESCUELA. 


\subsection{Subiendo fotografías de nubes y meteoros}

Esta es una actividad que intentamos continuamente estimular con recordatorios a los centros. Los alumnos identifican la fotografía y nos piden que evaluemos si están bien denominados la nube o el meteoro según la clasificación internacional que pueden consultar en el portal en fichas de trabajo que se han incorporado. Una vez pasado el filtro aparecen en el portal (figura 6) con el nombre del autor, colegio, localidad y nombre correcto de la nube o meteoro.
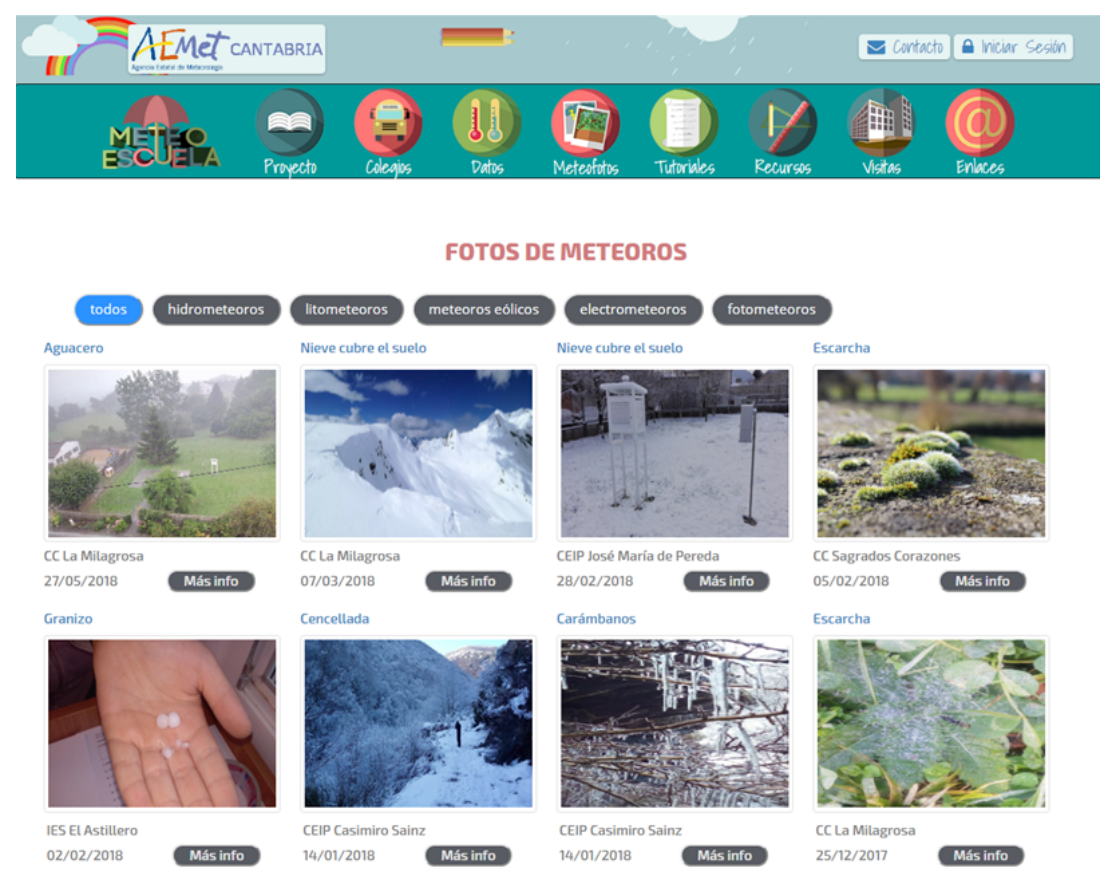

Figura 6. El apartado de nubes y meteoros.

Esta actividad se procura incentivar con la convocatoria a primeros de año de un concurso de fotografías (cada año se puede variar el lema), meteoros, etc. (el próximo se denominará «ciclo del agua»). El resultado del concurso se hace público a mediados de marzo de modo que se publican las fotografías presentadas y ganadoras, estando expuestas en nuestra delegación todo el año y las ganadoras son premiadas con un pequeño detalle que se entrega a los ganadores en los actos del Día Meteorológico Mundial (23 de marzo) y son entregados por el Delegado del Gobierno en Cantabria y la Consejera de Medio Ambiente de nuestra comunidad autónoma. El resultado final del concurso es la divulgación de dichas fotos en el calendario escolar oficial de la Consejería de Educación, que se distribuye a todos los centros escolares de Cantabria.

\subsection{Utilizando el portal web METEOESCUELA}

El portal se desarrolló inicialmente utilizando un gestor de contenidos (Drupal) a partir de un convenio con la Escuela Taller del Ayuntamiento de Camargo (Cantabria). Siguiendo las especificaciones dadas fue desarrollado por los alumnos de dicha escuela taller con diseños de dibujos propios de gran calidad que siguen siendo muchos de los empleados en la actualidad. Han pasado tres años y el diseño general ha variado algo, aunque manteniendo la filosofía inicial, además de haberse realizado por los propios medios informáticos de AEMET mediante el gestor de contenidos «October». El portal funciona con buen rendimiento en dispositivos móviles. La página web se aloja actualmente en servidores propios de la Agencia pues inicialmente se utilizaron durante un tiempo servidores del Gobierno de Cantabria. El diseño actual está realizado de forma que es fácilmente adaptable a cualquier otra delegación territorial que quiera adentrarse en METEOESCUELA. 


\section{CONCLUSIONES}

Este proyecto es fruto de una visión no plasmada en la práctica desde hace muchos años y que resultó muy costoso en sus inicios. Sin embargo, en la actualidad se encuentra en una fase de funcionamiento que permite implementarlo con relativa facilidad en cualquier otra delegación de AEMET.

Un mérito añadido es que se ha desarrollado e implantado con el trabajo extra, además de sus cometidos habituales de sus puestos de trabajo, de un grupo de trabajadores de la Delegación Territorial en Cantabria.

La página web se adapta fácilmente y los requisitos son que exista personal dispuesto a dar soporte a los centros escolares. Así como reciclar material de observación para una red pluviométrica.

Si se piensa en una red termopluvio habrá que buscar la colaboración de las consejerías de educación.

En un futuro sería deseable integrar METEOESCUELA con el proyecto MiniMET (http://es.minimet.net), un proyecto que propone la fabricación por los propios centros de una estación meteorológica automática y que, aún en fase piloto, es impulsado por el Departamento de Producción de AEMET.

\section{AGRADECIMIENTOS}

AEmiliano Guillén, antiguo becario de AEMET, por su inestimable ayuda en el arranque del proyecto, ya que fue el profesor de los jóvenes de la Escuela Taller de Camargo que diseñaron y elaboraron la primera versión del portal METEOESCUELA.

Un especial agradecimiento a los alumnos y coordinadores del proyecto en la Escuela Taller de Camargo, que llevaron más allá de su tiempo el trabajo en esta web: Sergio Palacio Ródenas, Raúl Pablos de la Prieta, Verónica Ruiz Antolín (alumnos) y Fernando Lobato (coordinador de la Escuela Taller VII).

A Jesús García y Sofía Jiménez, responsables de la Escuela Taller de Camargo.

Al Ayuntamiento de Camargo y al Centro de Investigación del Medio Ambiente (CIMA) del Gobierno de Cantabria que han continuado apoyando a METEOESCUELA desde el inicio.

A Leandro Morante, director del CIMA en los momentos iniciales, por su ayuda inestimable.

A la Dirección General de Innovación y Centros Educativos, a su Directora General M. ${ }^{a}$ Isabel Fernández así como a Carlos Cruz, Balbi García y Míriam González por continuar apoyando sin fisuras y con gran entusiasmo este proyecto. A Pilar Barquín, José Ángel Olavarría y Pepín Villasante, que estuvieron desde el principio apoyando con igual entusiasmo.

A Raúl Corredor, de AEMET, por su apoyo en cuestiones informáticas y a todos los compañeros de la delegación que, de una forma u otra, han apoyado y apoyan el proyecto.

Finalmente, a nuestro recordado Rafa Ancell, el meteorólogo de la delegación que siempre tuvo el sueño de este proyecto aunque no pudiera llegarlo a ver hecho realidad. 
